En ce qui concerne le fini des surfaces, les normes américaines spécifient un fini meulé au grain d'abrasif $n^{\circ}$ 150-180 (fini $n^{\circ} 4$ ). Quoiqu'il en soit, les travaux de O. W. KAUFMANN et al. (1960, $J$. Dairy Sci., 43, 28) et de Masurovsky et Jordan $(1958, J$. Dairy Sci., 41, 1342) ont prouvé que l'efficacité des traitements de nettoyage et de stérilisation des aciers inoxydables 18/8 est identique quel que soit le fini de la surface (fini $2 B, 3,4,7$ ). En général, on accorde une préférence au fini no 4 pour des raisons purement esthétiques.

\title{
GONGLUSION
}

L'emploi de l'acier inoxydable s'étant développé dans bien d'autres réalisations, et ses productions s'étant par ailleurs nettement améliorées, ce matériau est aujourd'hui beaucoup plus abordable et d'un prix compétitif. C'est pourquoi ce nouveau mode de ramassage devrait pouvoir être entièrement réalisé dans ce matériel sans pour cela être d'un prix plus élevé, et cependant d'un amortissement plus facile parce que de bien plus longue durée.

\section{L'ACTION DE LA PRÉSURE SUR LA CASÉINe (fin)}

\author{
par \\ Ch. ALAIS et P. JOLLÈS
}

\section{DISGUSSION}

\section{Le substrat de la présure dans la réaction primaire.}

Les résultats que nous avons obtenus soutiennent la conception de WAUGH et V. Hipper [21] selon laquelle l'attaque immédiate et rapide de la présure porte sur la caséine $x$, d'une manière spécifique. Cette réaction primaire se produit avec de faibles quantités d'enzyme et précède toujours la coagulation.

Ces résultats sont :

a) La formation d'une proportion élevée d'azote non protéique avec la caséine $\times$ (5 fois plus qu'avec la caséine entière);

b) L'identité de la composition en acides aminés des caséinoglycopeptides provenant de différentes caséines de vache : caséine entière, caséine $\alpha$ et caséine $x$;

c) L'identité des acides aminés C-terminaux dans ces caséinoglycopeptides ; 
d) La proportion élevée des glucides de la caséine $*$ se retrouvant dans le principal produit soluble de la réaction : le caséinoglycopeptide.

\section{La caséine $x$ de vache.}

La caséine $x$ doit être rangée dans le groupe des glycoprotéines, tel que le définit WINzLeR [22] : composés ayant principalement un caractère protéique et contenant plus de 1 p. 100 de glucides unis à la partie protéique par une liaison ferme.

Dans la partie glucidique de la caséine $x$ nous avons trouvé les 3 types moléculaires habituellement rencontrés dans les glycoprotéines et en proportions voisines : un sucre neutre : le galactose, un sucre aminé : la galactosamine et un sucre acide : l'acide $\mathrm{N}$-acétylneuraminique. On peut remarquer à ce propos que la caséine de vache ne contient pas d'acide $\mathrm{N}$-glycolylneuraminique contrairement à la caséine de chèvre et à la caséine de brebis.

MaRIER et coll. [23] estiment que tout l'acide sialique de la caséine entière provient de la caséine $x$ et que celle-ci est caractérisée par une teneur de 2,3 p. 100 . Ils ont analysé la caséine $x$ obtenue par quatre méthodes de purification et ont trouvé des teneurs en acide sialique très différentes les unes des autres. Ces auteurs considèrent que la teneur en acide sialique est un indice de pureté d'une préparation. La moyenne de nos déterminations est précisément de 2,3 p. 100 (tableau 5).

On peut comparer la caséine $x$ à la caséine $\alpha_{3}$, qui est le constituant insensible au calcium dans le fractionnement de McMeEKIN et coll. $[24, a]$. La composition en acides aminés de la caséine $\alpha_{3}(24,6)$ et celle que nous avons déterminée pour la caséine $x$ présentent de grandes similitudes, mais la dernière a un contenu plus élevé en hydroxy-aminoacides. En ce qui concerne la partie non peptidique, on constate des écarts notables; la caséine $\alpha_{3}$ est plus riche en phosphore $(0,35$ p. 100$)$ et plus pauvre en hexose $(0,75$ p. 100) et en acide sialique (1,2 p. 100) que la caséine $x$. Au sujet de la teneur en phosphore, il faut noter que, dans le cas de la caséine $x$, on peut la considérer comme un indice de pureté; plus la purification progresse, plus la teneur en phosphore diminue. C'est l'inverse de ce qui se produit avec l'acide sialique.

SwaIsGood et BrunNer [45] viennent de publier les résultats. d'une analyse de la caséine $x$; ils ont obtenus des valeurs proches des nôtres pour tous les acides aminés, sauf pour la cystine.

La caséine $x$ que nous avons examinée se caractérise par l'absence de groupe aminé terminal libre, pouvant être décelé par la 
méthode de Sanger [16]. Dans la caséine $\propto$ Wissmann et NitschMANN [54] ont caractérisé deux acides aminés N-terminaux : la lysine et l'arginine. Cependant Manson [55] n'a caractérisé que la lysine, dans une caséine $\alpha$ exempte de caséine $*$ (elle correspond à la fraction $\alpha_{S}$ de $W_{A U G H}$; on pourrait déduire de ce résultat que le résidu Arg terminal trouvé par les précédents auteurs provient de la caséine $x$ présente dans la caséine $\alpha$ qu'ils ont analysée. Nos résultats infirment cette hypothèse.

La caséine $x$ obtenue par la méthode de McKenzie et WAKE [11], que nous avons utilisée, apparaît homogène dans les examens d'ultracentrifugation, aussi bien à $p H$ 6,5 et $4^{\circ}$, qu'à $p H \quad 8,6$ et $20^{\circ}$ en solution d'urée [12] ; ces mêmes auteurs n'ont observé qu'une bande rapide dans l'électrophorèse en gélose et une bande étirée dans l'électrophorèse en gel d'amidon. Cependant, RibadeauDumas [26] a fait des réserves sur l'homogénéité de cette substance. Récemment BeEsy [25] a émis l'hypothèse que la caséine $x$ serait formée de 3 unités de même grandeur moléculaire, liées entre elles par des liaisons secondaires; tout l'acide sialique serait dans une unité et toute la cystine dans une autre.

Nous reviendrons plus loin sur le poids moléculaire de la caséine $x$.

\section{Le caséino-glycopeptide.}

La réaction primaire de la présure sépare de la caséine $x$ la substance soluble dans l'acide trichloracétique à 12 p. 100 et non dialysable, que nous avons appelé "Caséino-glycopeptide ". Sa composition a paru tout d'abord insolite par comparaison avec celle de la caséine dont il est issu. On peut considérer qu'il représente une réunion des composants de la caséine $x$ portant des groupes hydroxyles : thréonine, sérine et glucides. Pour les glucides, il s'agit peut-être d'une séparation totale?

On peut ranger le caséino-glycopeptide dans le groupe des " mucoïdes" [22], en raison de sa teneur élevée en galactosamine et en glucides totaux.

Le caséino-glycopeptide ne contient pas d'acides aminés aromatiques et soufrés, ni d'arginine (ces acides aminés sont relativement abondants dans la caséine $x$ ). La composition particulière de la partie peptidique du caséino-glycopeptide, que nous avons présentée, et la constance de cette composition d'une préparation à l'autre ont été également constatées par NITSCHMANN et coll. [27, 28] et par HaBERManN et coll. [29], pour des préparations provenant de diverses caséines de vache. 
Les auteurs précédents ont identifié les mêmes glucides que nous-mêmes, sauf HABERMANN et coll. [29] qui, dans une seule préparation sur quatre examinées, ont trouvé du xylose (à notre connaissance ce sucre n'a pas été décelé dans la caséine). Au point de vue quantitatif, des écarts importants existent entre les résultats de différentes déterminations des trois composants glucidiques; les méthodes analytiques utilisées ne peuvent à elles seules en rendre compte. Il est probable que les méthodes préparatives ont une influence sur la teneur en glucides.

Nous avons montré que le phosphore est lié à la sérine, que l'acide sialique est en position terminale et que les mêmes acides aminés C-terminaux se trouvent dans le caséino-glycopeptide et dans la caséine $x$. Le caséino-glycopeptide est certainement placé à une extrémité de la molécule de caséine $x$.

La partie glucidique présente des analogie avec les groupements prosthétiques de mucoïdes isolés de différents produits biologiques : mucoprotéine salivaire, orosomucoïde (ou glycoprotéine acide $\alpha_{1}$ ) du plasma sanguin, mucolipide cervical. Dans ces produits, l'acide sialique occupe la position terminale et est lié à un sucre neutre ou à un sucre aminé, lequel est rattaché à la chaîne peptidique qui forme la structure de base.

Un type de structure que l'on pourrait considérer comme l'inverse du précédent, peut également être envisagé pour le caséino-glycopeptide : chaîne glucidique à laquelle sont attachés des peptides. Un exemple bien connu est celui du glycopeptide des parois cellulaires de Staphylococcus aureus [57]; la chaîne est formée de $\mathrm{N}$-acétyl-glucosamine, dont un résidu sur deux porte le groupement lactyl (acide muramique). Un peptide composé de 5 résidus aminés est relié à chaque groupe lactyl. Ce type de structure semble-moins bien convenir que les précédents, dans le cas du caséino-glycopeptide. En effet l'acide sialique doit en grande partie occuper une position terminale; de plus nous n'avons pas pu caractériser d'acides aminés N-terminaux, dans les conditions habituelles.

\section{Le rôle de l'acide sialique.}

Il a été montré que l'acide sialique contrôle certaines propriétés physico-chimiques et biochimiques des mucoprotéines. D'après JEANLOz, il s'agit d'un des problèmes les plus importants dans l'étude de ce type de substance [30].

Les conséquences de la présence de l'acide sialique dans une molécule de mucoprotéine, en ce qui concerne certaines propriétés 
de cette dernière, ont été mises en évidence par GotTschalk et Thomas [31]; elles découlent de la forte charge négative du groupe carboxylique ( $\mathrm{pK} 2,6$ ). La répulsion électrostatique des groupes carboxyliques de l'acide sialique s'ajoute à l'effet des liaisons hydrogènes entre les groupements prosthétiques voisins pour maintenir la rigidité moléculaire, en dépit de la flexibilité potentielle aux endroits des liaisons par les résidus de proline. La molécule possède une forme allongée, avec un rapport axial élevé. Si l'on réduit l'ionisation de l'acide sialique, par abaissement du $\mathrm{pH}$, ou si on le détache par l'action de la neuraminidase, la molécule prend une forme compacte et les propriétés physiques de la mucoprotéine sont modifiées (chute de la viscosité des solutions dans l'exemple cité).

Le caséino-glycopeptide a une propriété importante : il ne traverse pas les membranes cellulosiques utilisées pour la dialyse. Cette propriété est probablement la conséquence de la forme de la molécule et de sa charge.

En ce qui concerne la caséine $\varkappa$, l'acide sialique joue peut-être un rôle dans sa propriété capitale : la stabilisation des constituants de la micelle de caséine entière ? D'après WAUGH [56], la caséine $x$ est la molécule la plus rigide du complexe et son aptitude à former, en interaction avec la fraction $\alpha_{S}$, des micelles stables serait en rapport avec cette rigidité moléculaire. Nous avons montré que la caséine $x$ est la fraction de beaucoup la plus riche en acide sialique (caséine entière 0,36 p. 100 , caséine $\times 2,3$ p. 100).

A ce point de vue, l'acide sialique facteur de rigidité, et les glucides facteurs de solubilité, peuvent être considérés comme formant le ou les groupements prosthétiques actifs de la caséine $x$. Lorsque la molécule est coupée en deux parties inégales, le caséinoglycopeptide se sépare et les propriétés du résidu, la paracaséine $x$, isolée ou associée aux autres fractions de la caséine entière, sont profondément modifiées, avec diminution de la solubilité et disparition du pouvoir stabilisant.

Récemment, Thompson et Pepper [32] ont montré que le pouvoir stabilisant de la caséine $\varkappa$, vis-à-vis de la caséine $\alpha_{\mathrm{S}}$, diminuait sensiblement après l'action de la neuraminidase. Ces auteurs pensent néanmoins que l'acide sialique lié ne suffit pas, à lui seul, à expliquer l'aptitude de la caséine $x$ à former des micelles avec la caséine $\alpha_{S}$ en présence de calcium.

\section{La liaison coupée par la présure.}

La question de l'activité protéolytique de la présure est débattue depuis longtemps. Holter [33,] dans des expériences minutieuses, 
n'a constaté, au cours de l'action de la présure ordinaire (non cristallisée) sur la caséine, qu'une très faible augmentation du nombre des groupes basiques, dépassant à peine les limites d'erreur de la méthode. Nitschmann et VARIN [34] ont repris ces mesures titrimétriques; ils ont utilisé la présure cristallisée à très forte dose (10 000 fois plus que dans nos expériences, en moyenne) et l'ont fait réagir sur la caséine entière. Après une durée d'action correspondant à celle de la coagulation, ils n'ont pas constaté une augmentation sensible du nombre de groupes acides et basiques.

Nous avons montré qu'après la réaction de la présure sur la caséine $x$, deux acides aminés C-terminaux apparaissent dans la paracaséine $x$, la leucine et la phénylalanine, que l'on ne peut déceler ni dans la caséine $x$ ni dans le caséino-glycopeptide. Par contre, nous n'avons pas pu mettre en évidence jusqu'à ce jour d'acide aminé $\mathrm{N}$-terminal dans l'autre produit de la réaction, le caséino-glycopeptide. Ces résultats semblent s'opposer à l'hypothèse de la coupure d'une liaison peptidique par la présure.

Par la méthode tritimétrique à $p$ H constant, GARnIer et coll. [41] ont montré qu'un très petit nombre de groupes ionisables apparaît après action de la présure sur la caséine $x$ le rapport étant un groupe pour $55000 \mathrm{~g}$ de caséine $x$. Ce groupe a un pK moyen compris entre 4 et 5 ; ce qui conduit à penser à une liaison du type ester dont le groupe ionisable serait un groupe carboxylique. Récemment, JoLlìs et coll. [15] ont étudié l'action réductrice de $\mathrm{LiBH}_{4}$ sur la caséine $x$ et ont observé la formation d'une substance soluble et d'une substance insoluble qui ressemblent au caséino-glycopeptide et à la paracaséine $x$. Le phénylalaninol a été détecté dans la partie insoluble et non pas le leucinol. Il se pourrait que la présure coupe une liaison ester entre le groupe carboxylique de la phénylalanine et un groupe $\mathrm{OH}$ appartenant au caséino-glycopeptide.

\section{La réaction primaire et la protéolyse non spécifique.}

La coupure de la liaison considérée ici intervient au cours de la réaction spécifique (réaction primaire). La cinétique de cette réaction peut être étudiée au moyen de la méthode que nous avons décrite : détermination en fonction du temps, de l'azote non protéique soluble dans l'acide trichloracétique à 12 p. 100 de concentration finale.

La réaction primaire s'apparente aux réactions de protéolyse spécifique limitée au cours desquelles une nouvelle espèce de protéine est formée par séparation d'un peptide. C'est notamment 
le cas de l'activation de proenzymes : pepsinogène, trypsinogène, chymotrypsinogène (voir en particulier DESNUELLE et ROVERY [35] La réaction de la thrombine sur le fibrinogène présente de grandes similitudes avec celle de la présure : la formation de la fibrine s'accompagne de la libération de deux fibrino-peptides [36]; la réaction est très rapide.

En dehors de la réaction primaire, nous avons mis en évidence une réaction de "protéolyse générale " que l'on peut suivre dans le temps en précipitant les protéines du mélange réactionnel par l'acide trichloracétique à 2 p. 100 [1]. Elle se poursuit à faible vitesse, dans les conditions de nos expériences, bien au-delà du temps nécessaire à la coagulation.

La réaction protéolytique de la présure sur différents substrats a été étudiée, notamment par BERRIDGe [37] sur l'hémoglobine; le $p \mathrm{H}$ optimum se situe entre 3,8 et 4,0 (alors que la pepsine, contenue dans les présures non purifiées, a dans ces conditions un $p$ H optimum d'action vers 1,8-2,0). Frsh [38] a trouvé le même $p \mathrm{H}$ optimum pour la présure réagissant sur la chaîne $\mathrm{B}$ de l'insuline ; il a montré que le mode d'action de la présure est voisin de celui de la pepsine et qu'elle agit sur un substrat de celle-ci : la carbobenzoxyglutamyltyrosine, alors qu'elle n'agit pas sur un substrat de la chymotrypsine : l'ester éthylique de l'acétyltyrosine et qu'elle n'a pas d'activité phosphamidasique.

Lorsqu'on étudie les activités enzymatiques de la présure, il faut done distinguer la réaction protéolytique non spécifique de la réaction primaire; cette dernière n'est peut-être pas une protéolyse, strictu senso.

\section{Les bilans analytiques et les poids moléculaires.}

Les résultats que nous avons obtenus par l'analyse de la caséine $x$ et des différents caséino-glycopeptides, en ce qui concerne les acides aminés, les sucres neutres, les sucres aminés, les acides sialiques et l'acide phosphorique, font apparaître un reste indosé, qui représente en moyenne 13 p. 100 de la substance sèche. Nous n'avons aucune indication concernant la présence éventuelle d'autres types de composants, en dehors de la possibilité du bloquage des groupes aminés terminaux par un acide organique.

Ces bilans analytiques déficitaires constituent une anomalie; mais une anomalie qui semble être la règle dans le domaine des glycoprotéines et des mucoïdes. Gotтschalk et Simmonds [39] écrivent : "un bilan complet des constituants d'une mucoprotéine riche en glucides semble actuellement irréalisable "; dans l'analyse 
de la mucoprotéine salivaire du mouton lequrs résultats ne rendent compte que de 88,7 p. 100 du poids total de la substance. Outre la destruction d'acides aminés au cours de l'hydrolyse chlorhydrique totale, ces auteurs indiquent deux autres causes de déficit:

1) Perte d'acides aminés par condensation irréversible avec des constituants glucidiques;

2) Pertes au cours de la détermination des sucres.

GHUYSEN [40] a souligné les difficultés que présente la détermination des sucres réducteurs dans un mucopeptide; une certaine quantité de sucres libérée au début de l'hydrolyse peut être ensuite détruite; cependant, après 3 heures d'hydrolyse, il reste encore du sucre qui échappe à l'analyse.

Il est vraisemblable que les valeurs indiquées aux chapitres précédents, pour les composants glucidiques, sont en dessous des valeurs réelles, par suite de la présence probable de ces composants dans l'indosé. L'erreur relative sur la partie peptidique, la plus abondante, doit être faible, d'autant plus que nous avons ten compte de la destruction partielle de la sérine et de la thréonine au cours de l'hydrolyse.

Si une partie du reste analytique était constituée par les glucides incomplètement dosés, l'erreur par défaut sur la proportion des composants glucidiques serait plus forte dans la caséine $x$, du fait de la proportion élevée de l'indosé (14 p. 100) et de la proportion assez faible des glucides (5,7 p. 100), que dans le caséino-glycopeptide où la teneur en glucides est beaucoup plus élevée (29 p. 100) et l'indosé plus faible que dans la caséine $\varkappa(9$ p. 100).

Nous avons vu que la molécule de caséine $x$ ne contiendrait pas assez d'acide sialique et de galactosamine, d'après les résultats du dosage direct, pour pouvoir donner une molécule de caséinoglycopeptide. Compte tenu de l'observation précédente, on peut supposer que la teneur réelle en glucides de la caséine $x$ est suffisante pour que cette condition soit satisfaite.

Les poids moléculaires minimums, calculés d'après la composition en acides aminés (caséine $x: 31800$, caséino-glycopeptide $x$ : 7800 ), sont en accord avec ceux qui correspondent à un résidu C-terminal (respectivement 32000 et 8300 ).

La détermination par les méthodes physiques (sédimentationdiffusion), effectuée par NitschmanN et coll. [42] sur une de nos préparations de caséino-glycopeptide, a conduit à admettre un poids moléculaire compris entre 6 et 8000 , donc du même ordre de grandeur que les valeurs ci-dessus. Par contre BRUNner et Tномpson [43] ont déduit, par les-mêmes méthodes, un poids 
moléculaire de 15300 , soit environ le double des précédents. Il pourrait s'agit d'un dimère. Leur produit a une teneur en phosphore relativement élevée : 0,6 p. 100 .

Pour la caséine $x$, McKenzie et WAKE [12] ont calculé un poids moléculaire approximatif de $26000 \pm 3000$, par la méthode de sédimentation-diffusion et la mesure de l'équilibre de sédimentation à $p \mathrm{H} \mathrm{12.} \mathrm{Cette} \mathrm{valeur} \mathrm{est} \mathrm{plus} \mathrm{faible} \mathrm{que} \mathrm{celle} \mathrm{que} \mathrm{nous} \mathrm{avons}$ indiquée : 32000 ; elle reste cependant du même ordre de grandeur Par contre, WAUGH [44] a calculé un poids moléculaire de 16000 , soit la moitié exactement de notre valeur. Ce poids de 16000 est incompatible avec la proportion de caséino-glycopeptide formé, de même qu'avec la composition de ce dernier et celle de la caséine $x$.

D'après les mesures titrimétriques à $p H$ constant, mentionnées plus haut [41], le poids moléculaire de la caséine $x$ devrait être de 55000 au moins, soit deux fois plus que celui donné par McKenzie et WaKe. Récemment, SWAISGOOD et BRunner [45] ont mesuré un poids moléculaire de 60000 pour le monomère de caséine $x$, au moyen de l'ultracentrifugation en présence d'agents dissociants.

\section{Action de la présure sur la caséine d'autres espèces.}

Peu de travaux scientifiques ont été publiés concernant la réaction de la présure sur la caséine d'autres espèces que l'espèce bovine. Nous avons montré que la présure détache des caséines de brebis et de chèvre des caséino-glycopeptides voisins, par leur composition, de celui qui est détaché de la caséine de vache. Il est probable que la présure réagit selon le même processus sur toutes les caséines des ruminants. Les différences spécifiques sont légères : d'une part les caséino-glycopeptides de brebis ou de chèvre contiennent de l'histidine, mais ne contiennent ni leucine, ni glycocolle; d'autre part ils contiennent l'acide $\mathrm{N}$-glycolylneuraminique en plus de l'acide $\mathrm{N}$-acétylneuraminique. A ce sujet il est curieux de constater que l'acide $\mathrm{N}$-acétylé se trouve presque pur dans la mucoprotéine salivaire du mouton (on l'a dénommé O-sialique : $O$ pour ovin), alors qu'il est en faible proportion, à côté de l'acide N-glycolyl dominant, dans la caséine ovine.

Nous avons montré antérieurement [46] que la caséine de brebis ne se comporte pas comme la caséine de vache, au cours de la réaction primaire; elle donne 1,5 fois plus de NPN que cette dernière. Nous avons cherché à isoler la caséine $x$ de brebis ; mais nous n'avons pas encore réussi à l'obtenir à l'état pur par la méthode de McKenzie et WAKE [11]; il ne se produit pas de précipitation par l'alcool et l'acétate d'ammonium, au stade de la puri- 
fication où se sépare la caséine $x$ de vache. Cependant, le produit que nous avons obtenu possède les propriétés caractéristiques de la caséine $x$, et il donne une proportion de NPN supérieure à celle que donne la caséine $x$ de vache. Il se pourrait que la caséine $x$ de brebis se distingue de la caséine $x$ de vache par une plus grande sensibilité à l'action de la présure.

\section{La présure et les autres enzymes coagulant le lait.}

Avec la pepsine on obtient une courbe d'azote non protéique à 12 p. 100 d'acide trichloracétique presque identique à celle de la présure [47]; mais la réaction cesse de se produire vers $p H$ 6,7 et au-dessus, alors que la présure est encore active (la pepsine ne coagule pas les laits frais dont le $p H$ est relativement élevé).

Cerbulis et coll. [48] ont comparé l'action de la pepsine à celle de la présure sur la caséine entière à $p H$ 6,4. Les deux enzymes produisent à peu près les mêmes effets, mais la présure donne moins de composants solubles à $p H$ 4,6 que la pepsine. Elle a une spécificité plus étroite que cette dernière. C'est également la conclusion des travaux de LINDQvist et STORgard [49].

HabermanN et coll. [29] ont préparé, par action de la pepsine sur la caséine entière de vache à $p H$ 6,8, un caséino-glycopeptide dont la composition est voisine de celle du caséino-glycopeptide préparé par action de la présure.

Par contre, la pepsine se différencie nettement de la présure par son $p H$ optimum d'activité dans la réaction de protéolyse générale, comme nous l'avons vu précédemment (présure : $p \mathbf{H} 4$, pepsine : $p \mathrm{H}$ 2). Elle s'en différencie également par l'intensité de la protéolyse. La pepsine produit, aux d'épens de la caséine $\alpha$, des peptides et des acides aminés ; alors que la présure ne produit que des polypeptides [50].

La présure et la pepsine se distinguent des autres enzymes pouvant coaguler le lait par le fait qu'elles donnent lieu à la réaction primaire, représentée par la courbe caractéristique NPN $f(t)$. Avec les autres enzymes, la réaction protéolytique générale se manifeste fortement en même temps et la réaction primaire est peu visible ou inapparente. Tel est le cas de la chymotrypsine, de la trypsine et de la papaïne, étudiées par MATTENHEIMER et NitschmanN [51]. C'est également le cas de différents enzymes étudiés par les chercheurs japonais $[52,53]$ : protéases de Bacillus et Pseudomonas; ficine, extraite du figuier; enzymes extraits de testicules de lapin, et d'autres viscères.

Etant donné que ces diverses protéases ont une activité pro- 
téolytique non spécifique élevée, par rapport à l'activité coagulante, leur emploi en pratique pour coaguler le lait conduit à des résultats différents de ceux que donne la présure.

En résumé, l'activité protéolytique non spécifique de la présure, dans les conditions normales de la coagulation du lait, à $p H$ 6,6-6,8, est très réduite. Par contre, l'activité enzymatique spécifique se manifeste rapidement dans la réaction primaire. Elle provoque la séparation du caséino-glycopeptide de la caséine $\%$, en ne coupant qu'un très petit nombre de liaisons principales. Cette modification limitée est suffisante pour faire perdre à la caséine $x$, transformée en paracaséine $x$, sa propriété essentielle de facteur stabilisant pour les constituants de la micelle de caséine, qui précipitent ensemble lorsque le milieu contient du calcium. Il est probable, d'autre part, que la séparation du caséino-glycopeptide démasque sur la paracaséine des groupes permettant l'établissement de liaisons secondaires nouvelles, liaisons qui assureraient la structure du réseau formant le coagulum.

\section{RÉSUMÉ}

La caséine $x$ de vache est une glycoprotéine contenant une partie non peptidique (6 p. 100) composée de galactose, galactosamine, acide sialique et acide phosphorique. Il reste une partie indosée, comme dans d'autres glycoprotéines connues.

La réaction primaire de la présure sur la caséine, que l'on met en évidence en déterminant l'azote non protéique libéré (soluble dans l'acide trichloracétique à 12 p. 100) aboutit à la séparation du casséino-glycopeptide. Cette réaction s'apparente aux réactions de protéolyse limitée comme celle de la thrombine sur le fibrinogène.

A partir de la caséine $x$ on obtient cinq fois plus de caséinoglycopeptide qu'à partir de la caséine entière de vache; mais, dans les deux cas, les substances ont la même composition en acides aminés et la même séquence C-terminale. Ces résultats confirment la conception du substrat spécifique, représenté par la caséine $x$.

Le caséino-glycopeptide est un mucoïde contenant de 20 à 30 p. 100 de glucides; il emporte la majorité des glucides de la caséine $x$ dont il provient. Son poids moléculaire est relativement élevé, environ 8000 . Sa composition en acides aminés est très différente de celle de la caséine $x$ ou de la paracaséine $x$; il contient beaucoup d'hydroxyamino-acides $(20$ p. 100) mais ni acides aminés soufrés et aromatiques, ni arginine. Les groupes acides sont en fort excès. La composition du caséino-glycopeptide explique sa 
grande solubilité. L'acide sialique joue probablement un rôle particulier dans les propriétés de la molécule.

On a mis en évidence les mêmes acides aminés C-terminaux dans la caséine $x$ et dans le caséino-glycopeptide (Thr, Val); après action de la présure, des acides aminés C-terminaux différents apparaissent dans la paracaséine $\times$ (Leu, Phe). Dans aucune de ces substances on n'a pu caractériser d'acides aminés N-terminaux, dans les conditions habituelles.

Les résultats qui se dégagent du présent travail permettent de développer de façon provisoire la formule de la caséine $\%$. Le caséinoglycopeptide est situé du côté C-terminal de la caséine $x$. Il est lié à la paracaséine $x$ par une liaison impliquant le résidu terminal (Phe) de cette dernière. Une liaison du type ester a été envisagée.

Lorsque la présure a réagi sur la caséine $x$, celle-ci perd ses groupements prosthétiques glucidiques, facteurs de solubilité et de rigidité moléculaire. Ce ce fait la paracaséine $x$ formée devient insoluble; elle est alors dépourvue de la propriété essentielle de la caséine $x$ : la stabilisation des autres composants des micelles, telles qu'elles existent dans le lait.

Les caséines de brebis et de chèvre donnent également lieu à la formation de caséino-glycopeptides, qui sont très voisins de celui de vache. La caséine de brebis a un comportement particulier.

\section{SUMMMARY}

The action of rennin on casein

\section{Part II. - Study of substrate and reaction products}

Cow's $x$-casein is a glycoprotein containing a non-peptidic part (6 p. 100) composed of galactose, galactosamine, sialic acid and phosphoric acid. There remains an undeterminated moiety (14 p. 100) as in a great number of other glycoproteins.

The specific " primary reaction " of rennin on easein is brought in evidence by determining the non-protein nitrogen soluble in 12 p. 100 trichloroacetic acid. This reaction gives rise to the caseinoglycopeptide; it is similar to other limited proteolytic reactions, as that of thrombine on fibrinogen.

The yield of caseino-glycopeptide is five times higher with $x$-casein than with cow's whole casein, but the two glycopeptides have the same amino-acid composition and C-terminal sequence. These results confirm the hypothesis that $x$-casein seems to be the specific substrate of rennin.

The caseino-glycopeptide is a mucoïd containing a sugar 
moiety of 20-30 p. 100 ; it contains nearly all the sugars of $x$-casein ; its molecular weight is about 8000 . The amino acid composition of the caseino-glycopeptide is very different from those of $x$-casein and para- $x$-casein; the content of hydroxyaminoacid is high and there are neither sulfur and aromatic amino acid nor arginine. The acidic groups are in excess. Such a composition explains the great solubility of the caseino-glycopeptide; sialic acid accounts for some properties of the molecule.

The same C-terminal amino acids have been detected in caseinoglycopeptide and in $x$-casein ( $\mathrm{Thr}, \mathrm{Val}$ ) ; after rennin action, new C-terminal amino acids appear in para- $x$-casein (Leu, Phe). No $\mathrm{N}$-terminal amino acids have been detected, in the usual conditions, in all these substances.

The results of this work allow us to indicate an hypothetical formula of $x$-casein. The caseino-glycopeptide, with nearly all the glucides, is located at the C-terminal side of the molecule; it is linked to para- $x$-casein through the C-terminal residue of this latter. An ester linkage has been proposed.

After rennin action, $x$-casein loses its glucidic prosthetic groups, which are factors of its solubility and its molecular rigidity. The para-x-casein formed in these conditions is insoluble and devoid of the essential properties of $x$-casein : stabilization of the other components in the micelles as they exist in milk.

The caseins of sheep and goat give also rise to caseino-glycopeptides which are very similar to that of cow, but the yield is higher with casein of sheep than with the two others.

\section{BIBLIOGRAPHIE}

[1] C. Alais, G. Mocquot, Hs. Nitschmann et P. Zahler. Helv. Chim. Acta, 36, 1953, 1955.

[2] S. Moore, O. H. Spackman et W. H. Stein. Anal. Chem. 30, 1958, 1185.

[3] P. Jollès, C. Alais et J. Jollès. Biochim. Biophys. Acta, 51, 1961, 309.

[4] C. Alais et P. Jollès. Biochim. Biophys. Acta, 51, 1961, 315.

[5] C. J. Rondle et W. T. J. Morgan. Biochem. J. 61, 1955, 586.

[6] L. Warren. J. Biol. Chem., 234, 1959, 1971.

[7] H. E. Sohulze, R. Sohmidtberger et H. Haupt. Biochem. Z., 329, $1958,490-507$.

[8] E. Bamann, E. Novotny et L. Rohr. Chem. Ber., 81, 1948, 438.

[9] P. J. Stoffyn et R. W. Jeanloz. Arch. Biochem. Biophys., 52, 1954, 373.

[10] E. Svennerholm et L. Svennerholm. Nature, 181, 1958, 1154.

[11] H. A. McKenzie et R. G. WAKe. Biochim. Bioph. Acta. 47, 1961, 240.

[12] H. A. McKenzie et R. G. WAKe. Austr. J.Chem., 12, 1959, 734.

[13] P. Jollès, C. Allais et J. Jollès. Arch. Biochem. Biophys., 98, 1962, 56. 
[14] N. J. Hipp, M. L. Groves, J. H. Custer et T. L. MoMeerin. J. Dairy Sci., 35, 1952, 272.

[15] P. Jollès, C. Alais et J. Jollìs. Biochim. Biophys. Acta, 69, 1963, 511.

[16] F. SANGer. J. Biochem., 39, 1945, 511.

[17] P. Edman. Acta Chem. Scand., 4, 1950, 283, 700.

[18] J. Jollès-Thaureaux, P. Jollès et C. Fromageot. Biochim. Biophys. Acta, 27, 1958, 298.

[19] J. Williams et F. Sanger. Biochim. Biophys. Acta, 33, 1959, 294.

[20] A. Gotsschalk. "The Enzymes ", Vol. IV, Academic Press, New-York, 1960,461 .

[21] D. F. WAUGH et P. H. v. Hippel. J. Am. Chem. Soc., 78, 1956, 576.

[22] R. J. Winzler. In "The plasma Proteins" (F. W. Putnam Edit.), Academic Press, New-York, 1960, 309-341.

[23] J. R. Marier, H. Téssier et D. Rosa, J. Dairy Sci., 46, 1963, 373.

[24 a] T. L. MoMeerin, N. J. Hipp et M. L. Groves. Arch. Biochem. Biophys., 83, 1959, 35 .

[24b] N. J. Hipp, J. J. Basch et W. G. Gordon. Arch. Biochem. Biophys., 94, 1961, 35.

[25] R. Bеeвy. J. Dairy Res., 30, 1963, 77.

[26] B. Ribadeau Dumas. Biochim. Biophys. Acta, 54, 1961, 400.

[27] Hs. Nitschmann et R. Henzi. Helv. Chim. Acta, 52, 1959, 1985.

[28] Hs. Nitschmann et R. Beeby. Chimia, 14, 1960, 318.

[29] W. Habermann, H. Mattenheimer, H. Sky-Peok et M. Sinohara. Chimia, 15, 1961, 339.

[30] R. W. Jeanloz. Bull. Soc. Chim. Biol., 42, 1960, 303.

[31] A. Gottschalk et M. A. W. Thomas. Biochim. Biophys. Acta, 46, $1961,91$.

[32] M. P. Thompson et L. Pepper. J. Dairy Sci., 45, 1962, 794.

[33] H. Holter. Biochem. Z., 255, 1932, 160.

[34] Hs. Nitschmann et R. Varin, Helv. Chim. Acta, 34, 1951, 1421.

[35] P. Desnuelle et M. Rovery, III' Congr. Intern. Biochim. Bruxelles $1955,78$.

[36] L. Lorand. Biochem. J., 52, 1952, 200.

[37] N. J. BerRidge. Biochem. J., 39, 1945, 179.

[38] J. C. Fisch. Nature, 180, 1957, 345.

[39] A. Gотtschalk et D. H. Simmonds. Biochim. Biophys. Acta, 42, $1960,141$.

[40] J. M. Ghuysen. Biochim Biophys. Acta. 50, 1961, 413.

[41] J. Garnier, G. Mocquot et G. Brignon. Comptes rendus, 254, 1962, 372.

[42] Hs. Nitschmann, H. Wissmann et R. Henzi. Chimia, 11, 1957, 76.

[43] J. R. Brunner et M. P. Thompson. J. Dairy Sci., 42, 1959, 1881.

[44] D. F. WAUgh. Faraday Soc. Discussions, 25, (1958), 186.

[45] H. E. Swatsgood et J. R. Brunner. Biochem. Biophys. Res. Commun., $12,1963,148$.

[46] C. Alais, H. Dutheil et J. Bosc. XVI ${ }^{\text {e }}$ Congr. Intern. Lait., vol. B 1962,643 .

[47] T. Tsugo et K. YAMAUChI. Bull. Agric. Chem. Soc. Japan, 24, 1960, 96. 
[48] J. Cerbulis, J. H. Custer et C. A. Zittle. Arch. Biochem. Biophys., 84, $1959,417$.

[49] B. Lindquist et T. Storgard. Acta Chem. Scand., 14, 1960,.1432.

[50] M. OHEDA. Japan J. Zootechn. Sci., 27, 1956, 81.

[51] H. Mattenheimer et Hs. Nitschmann. Helv. Chim. Acta, 38, 1955, 687.

[52] T. Tsugo. Japan Zootechn. Sci., 25, 1954, 6 et 26, 1955, 173.

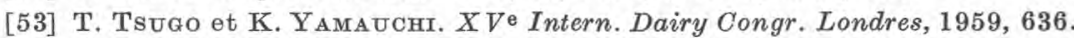

[54] H. Wissmann et Hs. Nitschmann. Helv. Chim. Acta, 40, 1957, 356.

[55] W. Manson. Nature, 184, 1959, 1393 et Arch. Biochem. Biophys., 95, $1961,336$.

[56] D. F. WAUGH. Abs. Pap. Am. Chem. Sac., april 1959, 49c.

[57] M. H. Mandelstamm et J. L. Strominger. Biochem. Biophys. Res. Comm., 5, 1961, 466.

\title{
SUPPLÉMENT TECHNIQUE
}

\section{L'EMPLOI DE L'ACIDE LACTIQUE ET DE SES DÉRIVÉS DANS LA FABRICATION DES ENCRES D'IMPRIMERIE ET DES PRODUITS DE SYNTHËSE}

\author{
par G. GENIN \\ Ingénieur E.P.C.I.
}

L'acide lactique est un produit connu depuis la plus haute antiquité et si nos ancêtres ne savaient pas l'isoler, par contre, ils l'utilisaient sous différentes formes. On a appris ensuite à isoler ce produit, à le préparer sous la forme de solution concentrée et, à différents états de pureté, il a été utilisé dans diverses industries: par exemple dans la préparation de produits pharmaceutiques sous la forme d'un acide de haute pureté devant répondre aux spécifications de la Pharmacopée ou, sous une forme moins pure, dans les industries alimentaires et également dans le tannage du cuir. Dans ces dernières applications, la présence dans l'acide utilisé de proportions plus ou moins importantes d'autres hydrates de carbone et de cendres était sans grande importance.

On ne peut done pas dire, pour cette catégorie d'utilisateurs, que l'acide lactique constitue une matière première nouvelle. Par contre, dans les industries chimiques et para-chimiques, la situation n'apparaît pas sous le même jour et l'emploi de cet acide commence à être envisagé, dans certaines branches de l'industrie, en particulier dans la fabrication des peintures, vernis et produits assimilés, soit directement, soit sous la forme de certains dérivés. Cette tendance résulte de la mise au point, il y a quelques 